\title{
Independent Assessment of the Major Nuclear Transient without Excessive Conservatism
}

\author{
Il S. Lee ${ }^{1,2}$, Min K. Cho ${ }^{1}$, Kyung L. Baek ${ }^{1}$, Yong C. Kim² \\ ${ }^{1}$ Korea Institute of Nuclear Safety \\ 62 Gwahak-ro, Yuseong, Daejeon, Korea, 305-338 \\ islee@kins.re.kr \\ ${ }^{2}$ Korea University \\ Department of Mechanical Engineering, 145 Anam-ro, Seongbuk-gu, Seoul, Korea
}

\section{Extended Abstract}

As concern on the nuclear safety is getting more increased by the public and it is raising safety issue on its transient events in worldwide, it has possibility highly to submit operating licensing or amendment as a type of realistic evaluation method for major transients. So, in order to prepare the future regulatory demands or needs, it is needed to develop the overall regulatory framework for realistic evaluation method on the safety review with respect to the accident analysis in Korea [1].

This study is to assess the main steam line break (MSLB) accident which is considered as significant transient according to regulatory guide. MSLB accident in a nuclear power plant (NPP) is the over-cooled transient with rapidly increasing steam mass flow to be caused asymmetric core temperature and mass flow. The main concerns are the possibility of the recriticality, and degradation of nuclear fuel cladding.

KINS have been used to evaluate MSLB with three different computational code such as system, CFD and sub-channel analysis code considering actual operation status without excessive conservatism.

Firstly, it has been analyzed system behavior of asymmetric events using the system code. The primary information is mass flow rate, temperature, and pressure in the cold and hot-leg, where it is essential to evaluate asymmetric phenomena in the core region while NPP is experiencing transient. Second, commercial CFD software calculated the distribution of temperature and mass flow rate in the core bottom based on the information from the results of system analysis. This step has been able to identify distribution of the 3-dimensional coolant in the core bottom. So, it plays a key role in making a decision how to model it into sub-channel analysis. Finally, sub-channel analyzer for departure from nuclear boiling ratio (DNBR) has been precisely evaluated critical heat flux, and it was also used to evaluate the thermal margin based on the asymmetric mass flow rate and temperature [2]. This paper focused on the results of system code because it was most important to understand the system response in independent regulatory assessment.

Steady and transient calculation were successfully performed using system code after modifying the hydraulic component and control logic considering the actual operation condition of the NPP. Calculation results showed large asymmetry in pressure and inventory level between steam generators. This calculation was also showed the degree of the safety margin from the evaluation of the DNBR which is one of the important acceptance criteria in nuclear safety law of Korea.

\section{References}

[1] I. S. Lee, D. H. Yoon, T. H. Kim, Y. C. Kim, "Regulatory Code System Configuration for DNBR on the Asymmetric Events with High Burn-up Fuel Issues," in Transactions of the Korea Nuclear Society, Jeju, Republic of Korea 2019.

[2] I. S. Lee, D. H. Yoon, T. H. Kim, Y. C. Kim, "Development of Regulatory Audit Framework for Asymmetric Events of the Non-LOCA," in Transactions of the American Nuclear Society, Minneapolis, USA 2019. 